\title{
Oculomotor apraxia and disrupted sleep with nocturnal ballistic bouts in ADCY5-related
} disease

Bettina Balint, $\mathrm{MD}^{1,2^{*}}$, Elena Antelmi, $\mathrm{MD}^{3^{*}}$, Niccolo E. Mencacci, MD, PhD, ${ }^{4}$ Amit Batla, $\mathrm{MD}^{1}$, Sofia Eriksson, ${ }^{5}$ Matthew Walker, ${ }^{5}$ Adolfo M. Bronstein, FRCP, 7,6 Kailash P. Bhatia, FRCP ${ }^{1}$

${ }^{1}$ Sobell Department of Motor Neuroscience and Movement Disorders, UCL Institute of Neurology, Queen Square, London, UK.

${ }^{2}$ Department of Neurology, University Hospital Heidelberg, Heidelberg, Germany

${ }^{3}$ Department of Biomedical and Neuromotor Sciences, Alma Mater Studiorum, University of Bologna, Bologna, Italy

${ }^{4}$ Northwestern University, Evanston

5

${ }^{6}$ Division of Brain Sciences, Imperial College London, Charing Cross Hospital, London, UK

${ }^{7}$ Department of Neuro-otology, The National Hospital for Neurology and Neurosurgery,

London, UK.

*)joint first/contributed equally

Corresponding author:

Prof Kailash Bhatia

Sobell Department of Motor Neuroscience and Movement Disorders

UCL Institute of Neurology

Queen Square

London WC1N 3BG UK

Email: k.bhatia@ucl.ac.uk

Telephone: +4402034488723

Word count: 1256 fallowance: 1250 )

Word count abstract: / 250 words

Number of references: 8

Number of tables: 1

Number of figures: 1

Supplemental Data: videos

Authors Disclosures: The authors report no disclosures related to the current manuscript. Full financial disclosures are listed at the end of the manuscript. 


\section{Abstract}

Objective: To characterise the distinctive eye movement disorder and the sleep-related dyskinesia in

Adenylate cyclase 5 (ADCY5) related disease.

Methods: Case series with formal eye movement examination and video-polysomnography.

Results: All three patients had an eye movement disorder characterised by oculomotor apraxia with gaze limitation most prominently in the vertical plane. All patients had disrupted sleep architecture with reduced sleep efficiency due to frequent and prolonged arousals and awakenings in the context of dyskinesia, which could arise from any sleep stage. The nocturnal movements could last up to 30 minutes and surpassed in their severity those seen during day-time.

Conclusion: The characteristic, apraxic eye movement disorder may help to distinguish ADCY5-

related disease from other childhood-onset hyperkinetic movement disorders. Similarly, the nocturnal exacerbations of dyskinesia ("ballistic bouts") seem to be a characteristic feature of the disease, affect the quality of life of patient and therefore require awareness and symptomatic treatment approaches.

Adenylate cyclase 5 (ADCY5) gene mutations have been recently recognised as the cause of a pleiomorphic childhood-onset hyperkinetic movement disorder, consisting mainly of chorea. ${ }^{1-3}$

The increasing number of reports indicate that the disease is not uncommon, and that the clinical spectrum expands to axial hypotonia, dystonia, myoclonus, and episodic worsening of dyskinesia. ${ }^{2,4}$ Hence, depending on the predominant movement disorder phenotype, this disorder can come into the differential diagnosis of early-onset chorea, such as that related to that due to TITF gene mutations, or myoclonus-dystonia due to epsilon sarcoglycan gene mutations, amongst others.

We have noticed that a distinct eye movement disorder as well as nocturnal exacerbations of movements may be useful clues in the differential diagnosis, and here present the results of formal eye movement examinations and the distinct video-polysomnographic features of our patients with genetically proven ADCY5-related disease. 


\section{Methods:}

The three patients underwent clinical examination, formal eye movement examination carried out by an eye movement expert (AB) and an overnight polysomnography (PSG) with standard montage, including conventional EEG, bilateral EOG, submentalis and anterior tibialis EMG, respiratory parameters, electrocardiogram and concomitant PSG-synchronized infrared video. Sleep stages were scored according to the current criteria (American Academy of Sleep Medicine 2014) by a registered and expert technician. ${ }^{5}$ Stages containing abnormal movements were marked and reviewed by clinicians with expertise in sleep medicine (SE, $A E, M W)$.

The study was approved by the local ethics committee, and all patients gave written informed consent.

\section{Results:}

Clinical and genetic details of these cases have been reported previously ${ }^{1}$ and are summarised together with clinical course and the current examination findings in the table.

\section{Predominantly vertical saccadic initiation failure and hypometria suggest oculomotor apraxia in ADCY5-related disease}

The details of the individual eye movement examinations are listed in the table. All three patients had difficulties initiating and executing full-range saccades, more pronounced in the vertical plane. This improved with head thrust or by using an audiovisual target, suggesting an apraxic type of eye movement disorder. Two of three patients displayed also gaze impersistance. None of the patients had spontaneous, gaze-evoked or positional nystagmus, or any other other cerebellar eye signs.

\section{Poor sleep efficiency, disrupted sleep architecture and nocturnal ballistic bouts in ADCY5-related disease}

The details of the individual PSG findings are listed in the table. In all patients, nocturnal PSG revealed disrupted sleep architecture (see hypnogramm of patient \#1, Fig. 1A) with severely affected sleep efficiency (SE), due to increased wake after sleep onset. Awakenings were usually associated with, or preceded by, snoring, hypopnea (Fig. 1B) or jerks involving the head or the limbs. They were then followed by attacks of severe and generalized chorea lasting from few minutes up to 30 minutes with discrete on- and off-set, in absence of clear epileptic discharges. The severity of the movements was worse at night compared to those observed during the daytime (see video). All patients showed sporadic periodic limb movements, while physiological atonia was present during REM sleep.

\section{Discussion:}

Here we describe three patients with genetically confirmed ADCY5 mutations, all of whom show an eye movement disorder and exacerbations of the movement disorder after arousal and/or awakening from sleep.

Eye movement abnormalities were that of she mainly the features of oculomotor apraxia, more pronounced in the vertical plane. Although, apraxic disorders, by definition, are also supranuclear, the difference with conventional supranuclear gaze palsies as in the Steele-Richardson's syndrome is that here the apraxic eye disorder improves with head thrusts or with reinforced sensory stimulation (as in patient \#2) and, critically, that saccadic velocities are well preserved.

Apart from These characteristics suggest an the apraxic type of eye movement disorder, there were also which would be in line with other clinical features of limb or orofacial apraxia in fcases \#2 and 
\#3, (see table). This suggests Most likely, the neuroanatomical basis would be a fronto-basal ganglia dysfunction, ${ }^{6}$ and indeed, this-would be consistent with prominent $A D C Y 5$ expression in frontal cortex and the basal ganglia.

Eye movements have not been detailed in the various reports of patients with ADCY5 mutations, except one report by Chang et al. ${ }^{2}$ They found that abnormal saccades in 5 of 6 patients. Four had absent saccadic upgaze, one patient had prolonged vertical saccadic latencies, and one patient was initiating horizontal saccades with a head thrust.

Putting these cases together with ours would indicate that apraxic eye movement abnormalities, particularly in the vertical plane, might be a characteristic feature of ADCY 5 mutations and help in the differential diagnosis from other causes of childhood-onset hyperkinetic disorders with autosomal dominant inheritance, such benign hereditary chorea due to TITF1 mutations.

Oculomotor apraxia can of course be seen in some other disorders that can present with chorea/dystonia such as ataxia telangiectasia and related disorders, including ataxia with oculomotor apraxia type 1-4. A vertical supranuclear gaze palsy is also present in Niemann Pick type $C$, where patients however exhibit a deficit in voluntary and reflexive vertical saccades with defects usually in speed and range of movements. ${ }^{7}$ Lastly, however, all of these are autosomal recessive conditions, which would still come into the differential diagnosis of de novo ADCY5 mutations.

Sleep-related exacerbation of dyskinesia has lately emerged as a specific feature of ADCY5-related movement disorder. ${ }^{2}$ Usually, sleep is a state of motor quiescence and, almost all movement disorders improve during sleep, the exception being of course sleep-related movement disorders. Nocturnal occurrence of movements, indeed, leads firstly to the suspicion of a parasomnia or of a seizure. Video-PSG represents the gold standard to assess such phenomena, but so far, sleep has been hardly investigated in the reported cohorts of patients affected with this disorder.

Chen et al firstly highlighted a worsening of the dyskinesia in ADCY5-related movement disorder during night-time. ${ }^{3}$ So far, there is a single paper reporting on sleep features investigated by means of PSG in a patient with ADCY5-related disease and documenting affected SE due to the persistence and exacerbation of dyskinesias, emerging during arousals from light sleep, mainly related to a mild hypopnea. $^{2}$

Our study confirms the finding of a disrupted nocturnal sleep due to the exacerbation of movements. Moreover, our PSG findings goes further documenting the persistence of the dyskinesia even during slow wave sleep. Interestingly, as reported in the patient by Chang et al., ${ }^{2}$ awakenings are associated with sleep-related jerks (myoclonus involving the head or the body) or with snoring or hypopnea, in absence of obstructive sleep apnoea. This is of interest, as it seems that disrupted nocturnal sleep is triggered by the re-emergence of the movement disorder itself and/or by hypopnea, that might hypothetically relate to dyskinesia involving the respiratory muscles. This latter point therefore needs to be further investigated in the future, by adding EMG leads over thoracic and abdominal muscles. Yet, another explanation can be that these patients might have a tendency to an increased arousal index, related to a dysfunction of sleep regulation itself. Adenylate cyclase 5 indeed in highly expressed in the nucleus accumbens, ${ }^{8}$ which is supposed to control the level of cortical arousal during the sleep-wake cycle, but also to promote motor activity. ${ }^{9}$

For patients, the episodic exacerbations of dyskinesia and the disrupted sleep can severely affect the quality of life. Particularly patient \#1, also otherwise clinically most severely affected, considered the nocturnal worsening of dyskinesias and lack of sleep as his main problem, and found Clonazepam $0.5 \mathrm{mg}$ nocte beneficial.

In summary, we here report characteristic eye movement abnormalities, characterised by apraxia with gaze limitation in the vertical plane, which may help to guide the differential diagnosis and 
Balint, Antelmi et al. 5

allows distinguishing ADCY5-related disease from other childhood-onset hyperkinetic movement disorders. Similarly, nocturnal exacerbations of dyskinesia seem to be a specific feature of the disease and may require symptomatic treatment approaches. 


\section{References}

1. Mencacci NE, Erro R, Wiethoff $S$, et al. ADCY5 mutations are another cause of benign hereditary chorea. Neurology 2015;85:80-88.

2. Chang FC, Westenberger A, Dale RC, et al. Phenotypic insights into ADCY5-associated disease. Movement disorders : official journal of the Movement Disorder Society 2016;31:1033-1040.

3. Chen YZ, Friedman JR, Chen DH, et al. Gain-of-function ADCY5 mutations in familial dyskinesia with facial myokymia. Annals of neurology 2014;75:542-549.

4. Carecchio M, Mencacci NE, lodice A, et al. ADCY5-related movement disorders: Frequency, disease course and phenotypic variability in a cohort of paediatric patients. Parkinsonism \& related disorders 2017;41:37-43.

5. Berry RB, Brooks R, Gamaldo CE, Harding SM, Marcus C, Vaughn B. The AASM manual for the scoring of sleep and associated events. Rules, Terminology and Technical Specifications, Darien, Illinois, American Academy of Sleep Medicine 2012.

6. Leigh RJ, Zee DS. The Neurology of Eye Movements: Oxford University Press, 2015.

7. Salsano E, Umeh C, Rufa A, Pareyson D, Zee DS. Vertical supranuclear gaze palsy in NiemannPick type $C$ disease. Neurological sciences : official journal of the Italian Neurological Society and of the Italian Society of Clinical Neurophysiology 2012;33:1225-1232.

8. Matsuoka I, Suzuki Y, Defer N, Nakanishi H, Hanoune J. Differential expression of type I, II, and $\mathrm{V}$ adenylyl cyclase gene in the postnatal developing rat brain. Journal of neurochemistry 1997;68:498-506.

9. Lazarus M, Chen JF, Urade Y, Huang ZL. Role of the basal ganglia in the control of sleep and wakefulness. Current opinion in neurobiology 2013;23:780-785. 
Figure 1:

Hypnogram (A) and sleep recording (B) of patient \#1. (A) The hypnogram shows markedly disrupted sleep architecture with frequent arousals, periodic limb movements of sleep, and occasional hypopnea. (B) The sleep recording shows an episode of hypopnoea followed by marked dyskinesia.

A
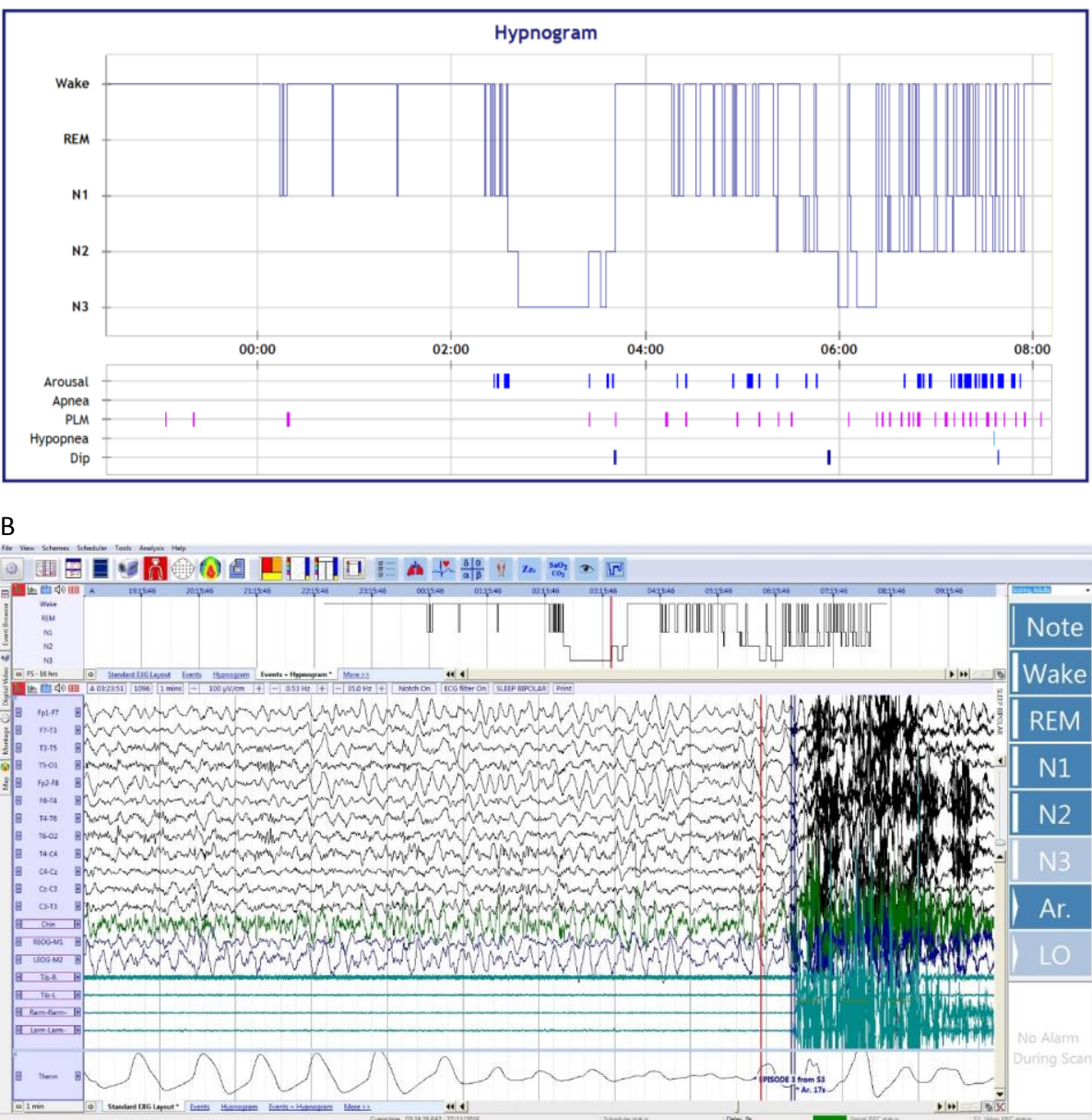

\section{Supplementary material:}

Videos.

Video 1: Comparison of day-time status and sleep-related bouts of chorea in patient \#1 and \#2. Segment 1 shows mild chorea in patient \#2 during day-time, contrasting with the severity of a ballistic bout of dyskinesia on PSG recording. Segment 2 shows patient \#1 with chorea worsening on action during day-time, and a much more severe, ballistic exacerbation, initiated by a shoulder jerk, of the chorea during PSG. Segment 2 shows the eye movements of patient \#1, with gaze impersistance 
interfering with e.g. during smooth pursuit, and hypometric saccades particularly in the vertical plane, with delayed initiation and often initiated by head thrust.

Video 2: Eye movement examination and other features suggestive of an apraxic disorder. Segment 1 shows the eye movement examination pf patient \#2 with hypometric saccades and long latencies, particularly pronounced on down gaze. Segment 2 shows striking difficulties copying gestures in the same patient.

\section{Author roles:}

1. Research project: A. Conception, B. Organization, C. Execution;

2. Statistical Analysis: A. Design, B. Execution, C. Review and Critique;

3. Manuscript Preparation: A. Writing the first draft, B. Review and Critique

Bettina Balint: 1A, 1B, 1C, 3A

Elena Antelmi: 1A, 1B, 3A, 3B

Niccolo E. Mencacci: 1C, 3B

Amit Batla: 3B

Sofia Eriksson: 1C, 3B

Matthew Walker: 1C, 3B

Adolfo M. Bronstein: 1C, 3B

Kailash P. Bhatia: 1A, 3A, 3B

\section{Acknowledgment:}

Bettina Balint is supported by the EAN research fellowship programme and the Robert Bosch Foundation.

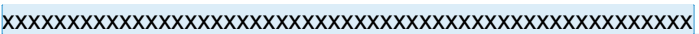

\section{Full financial disclosures:}

Bettina Balint is supported by the EAN research fellowship programme and the Bosch Foundation, and declares no conflicts of interest.

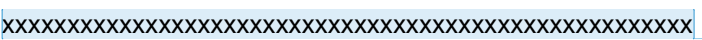

Kailash $P$. Bhatia has received grant support from Welcome/MRC, NIHR, Parkinsons's UK and EU Horizon 2020. He has received royalties from publication of the Oxford Specialist Handbook

Parkinson's Disease and Other Movement Disorders (Oxford University Press, 2008, 2016) and of Marsden's Book of Movement Disorders (Oxford University Press, 2013). He has received honoraria/personal compensation for participating as consultant/scientific board member from Ipsen, Allergan, Merz and honoraria for speaking at meetings and from Allergan, Ipsen, Merz, Sun Pharma, Teva, UCB Pharmaceuticals and from the American Academy of Neurology and Movement Disorders Society. 